\title{
MISSION PLANNING AND ORIENTATION MEASUREMENT FOR
}

\section{UAVs}

\author{
Sridurga Kasukurthi ${ }^{1}$, P. Krishna Chaitanya ${ }^{2}$ \\ ${ }^{1}$ Department of Electronics and Communications, Vignan's University Guntur, India \\ ${ }^{2}$ Department of Electronics and Communications, Vignan's University, Guntur, India
}

\begin{abstract}
The focus of this paper is to describe the orientation measurement of the multirotors using the raw data generated by the copter after Autonomous Flight. At first basic properties of multicopter is described. To attain the orientation measurement and attitude control of a copter, rotation matrices and body fixed frame position in earth frame are required. According to the requirements for orientation measurement of UAVs, the paper mainly concentrates on automated mission planning and processing as well as mission analysis. Here the following aspects are described in detail. (i) Mission requirements (ii) The automated mission generation and automated mission profile for the on board system (iii) Mission accomplishment on the field and the onboard recorded flight data analysis. Using the raw data generated by UAVs during the automated mission implementation, the orientation in the form of angular acceleration and angular velocity of a rigid body which is subjected to external forces like Gravity, Air Friction, Thrust, Torque has implemented in simulink. The flight Dynamics of a copter is described in detail to measure the Angular acceleration and Angular Velocity of the copter. Automated Mission implementation plays a major roll where the behaviour of the copter can be observed at each and every point of the flight.
\end{abstract}

Keywords: Orientation Measurement, Rotation Matrices, Automated Mission Planning, Mission Planner, Aurdu Pilot Mega2.6 Controller, Automation, Unmanned Aerial Vehicles

\section{INTRODUCTION}

Unmanned Aerial Vehicles (UAVs) are defined as a powered aerial vehicles they does not carry human operators, uses aerodynamic forces to perform lift operation. It can fly autonomously or it can be piloted remotely. An UAV can be expendable or recoverable, and can carry payloads. An UAV is capable of solving specific tasks like traffic surveillance, meteorological surveillance, archaeological surveillance, carrying specific payloads like couriers or solving tasks where presence of human pilot can be unhealthy or dangerous. In comparison with manned aerial vehicles, UAV is the one which has some advantages like low cost, longer endurance, less risk to the crews. As a part of UAVs hexacopter have the advantages that they can fly at high altitudes, in obstacles prone zones.

In recent years, UAV controlling technologies are progressing regularly. The main controlling methodologies are radio remote and preprogrammed. If communication link is not reliable, remote control modes no long has any effect, which leads to bread away from communication link and then UAV cannot update mission when planned or treat situations are changed. Even by combining the two methodologies, it is not possible to solve such problem in ambiguous situations. Autonomous control systems are used to perform well even in uncertain situations in system and environment for more period of time. Autonomous control systems can compensate for system failures without any human intervention. Artificial intelligence techniques are used to achieve autonomy.
This paper measures the orientation of the hexacopter in the form of Angular Accelerations and Angular Velocities using rotation matrices and body fixed frame position in earth frame which can be extracted from the analyzed data logs. The data logs are generated during automated mission implementation.

The mission is planned and loaded into the board (APM 2.6), and then data logs which have generated during mission implementation are downloaded from the board and analyzed to extract required data to measure the angular acceleration and angular velocity of hexacopter.

\section{AUTONOMOUS CONTROLL OF UAV}

Autonomous means not subject to control from outer controllers. An autonomous controller has ability to govern itself in the performance of control functions. Here automation is defined as operating the vehicle even in unstructured environment associated with uncertainty with the absence of human intervention[4].Within a limit an autonomous controller can deal with unpredictable situations, new tasks when system is in action and failures. The challenge of autonomous control is to optimize with ambiguous situations in real time without human intervention.

The difference between the automatic control and autonomous control is interpreted as an automatic control takes inputs from human and an autonomous control acquires inputs by their own. However the role of 
autonomous control is to do more than an automatic control. Autonomous controllers can be used in the areas like Manufacturing, Automotive, Food and beverage, Hospital and warehousing.

\section{MISSION PLANNING}

A Mission analysis that pursues required courses of action by enumerating a sequence of tasks, interpreting the supply to task distribution, and a time line for all task activities comprises a mission plan for autonomous controlling of a vehicle it is obligatory to have a platform. Here in this project we are using an open source application called Mission planner. Mission Planner is a full recommended ground control station application for Autopilot [2]. It can also acts as a ground control station for Plane, copter and Rover from where we can monitor the vehicle when it is in flight [7]. It is compatible only with Windows. Mission Planner can be used as a configuration utility, as a dynamic control supplement for your autonomous vehicles.

The series of things we need to do with mission planner:

- Loading the firmware into the autopilot (APM 2.6) which controls the vehicle

- Setting up, configuring and tuning the vehicle for optimum performance.

- Planning, saving and loading autonomous mission in APM by simply placing waypoint on Google map.

- Downloading and analyzing mission logs created by the vehicle.

- With appropriate telemetry hardware you can monitor the vehicle's status while in operation.

- With telemetry we can record telemetry logs which contain more information than the on-board autopilot logs, we can analyze logs and can operate the vehicle in FPV.

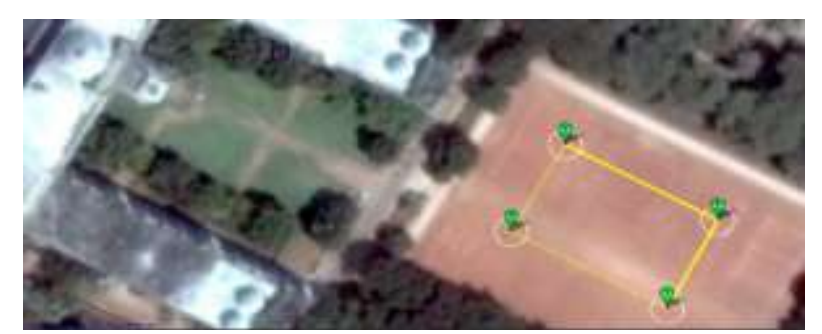

Fig -1: Planned mission for autonomous flight in our college premisis

\section{FLIGHT DYNAMICS}

The multirotor used in this paper is Hexacopter (F550) in which six arms connected symmetrically to central hub. The six propeller vehicle is controlled by pitching the main propeller. The electronics used for communication and control are integrated on chip which acts as a central hub together with battery. The source of the thrust of a hexacopter is located outside the centre of gravity. The rotation of rotors also produced a reaction torque opposite of direction of the rotation [3]. Since half of the propellers are spinning in one direction, the net torque when all rotors have equal speed is zero. The four basic movements for UAVs are throttle, pitch, yaw and roll. The values are extracted from the analyzed data logs, which has downloaded from the integrated board after flying. These values are used to define the orientation of the hexacopter in the form of angular acceleration and angular velocities [1].The Orientation and attitude of a hexacopter is described by Flight dynamics [8]

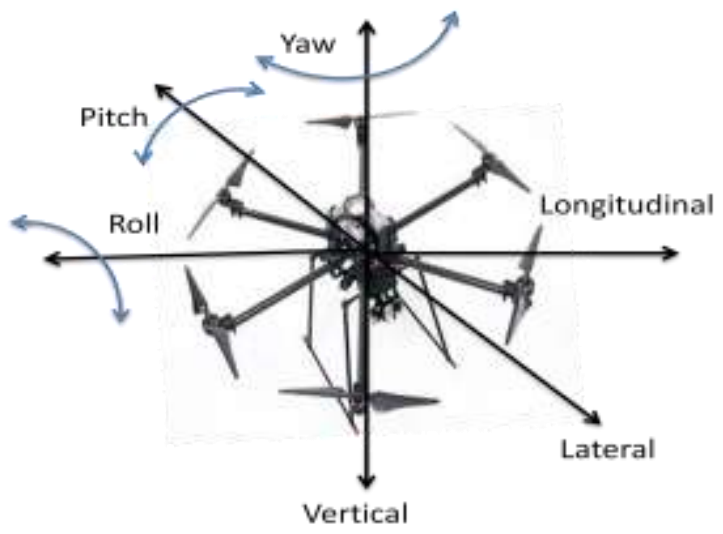

Fig -2: Six degrees of freedom of hexacopter

Here the control system of hexacopter is divided into inner loop and outer loop. Inner loop controls attitude and height while the outer loop controls the position where the position is controlled by sending reference values to the inner controller. Here in this project the outer loop is control system running on board. The task of the outer loop is to make hexacopter to follow the waypoints from a navigation system.

The flight board used is the Aurdu pilot Mega 2.6 developed by UAV Europe. Central processing unit of the board is used for communication, controlling air vehicle and collects data from sensors and filter the data. The board also contains a modem to communicate with a ground station. APM 2.6 consists of a Gyroscope which can measure attitude and body heading, Accelerometer which can measure Body heading, GPS which can give information about Latitude, Longitude, Flight direction and Flight speed, Barometer which is used to Measure Altitude, Altitude change. Let $\mathrm{E}=\mathrm{f}\left\{x_{\mathrm{E}}, y_{\mathrm{E}}, z_{\mathrm{E}}\right\}$ and a body fixed frame origin on the surface of the earth. As convenient when describing aircraft motion, the earth fixed frame is a north-east-down system with $x_{\mathrm{E}-}$

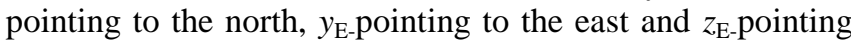
downwards. The body fixed frame's position in the earth fixed frame can be described by the vector $\xi=\left[\begin{array}{lll}X & Y Z\end{array}\right]^{\mathbf{T}}$ and its orientation, attitude and heading, by the vector $\boldsymbol{\eta}=\left[\begin{array}{lll}\boldsymbol{\theta} & \boldsymbol{\psi}\end{array}\right]^{\mathrm{T}}$ [5]. The total rotational matrix, to transform any quantity from earth frame to body frame, is obtained by [6]

$$
=\left[\begin{array}{ccc}
\mathrm{c} \theta \mathrm{c} \psi & {\left[\begin{array}{l}
x_{B} \\
y_{B} \\
z_{B}
\end{array}\right] \mathrm{c} \theta \mathrm{s} \psi} & -\mathrm{s} \theta \\
\mathrm{s} \phi \mathrm{s} \theta \mathrm{c} \psi-\mathrm{c} \phi \mathrm{s} \psi & \mathrm{s} \phi \mathrm{s} \theta \mathrm{s} \psi+\mathrm{c} \phi \mathrm{c} \psi & \mathrm{s} \phi \mathrm{c} \theta \\
\mathrm{c} \phi \mathrm{s} \theta \mathrm{c} \psi+\mathrm{s} \phi \mathrm{s} \psi & \mathrm{c} \phi \mathrm{s} \theta \mathrm{s} \psi-\mathrm{s} \phi \mathrm{c} \psi & \mathrm{c} \phi \mathrm{c} \theta
\end{array}\right]\left[\begin{array}{l}
x_{E} \\
y_{E} \\
z_{E}
\end{array}\right]
$$


The linear velocity of the hexacopter expressed in the body frame $\mathrm{V}=\left[\begin{array}{lll}u & v & w\end{array}\right]^{\mathrm{T}}$. The second time derivative of the position $\xi$ is

$$
\dot{\xi}=R_{L}^{T} V \quad \rightarrow(2)
$$

In order to relate the change of attitude with the body angular velocities the different steps of the rotation have to be considered. First, denote the body angular velocities $\boldsymbol{\omega}=[\boldsymbol{p} \boldsymbol{q} \boldsymbol{r}]^{\mathbf{T}}$, where $p$ is rotation around the $x^{\mathrm{B}}$-axis, $q$ is rotation around the $y^{\mathrm{B}}$ axis and $r$ is rotation around the $z^{\mathrm{B}}$ axis.

$$
\left[\begin{array}{l}
p \\
q \\
r
\end{array}\right]=\left[\begin{array}{ccc}
1 & 0 & -\sin \theta \\
0 & \cos \phi & \sin \phi \cos \theta \\
0 & -\sin \phi & \cos \phi \cos \theta
\end{array}\right]\left[\begin{array}{c}
\dot{\phi} \\
\dot{\theta} \\
\dot{\psi}
\end{array}\right] \rightarrow(3)
$$

Then the time derivate of the hexarotor's attitude $\boldsymbol{\eta}$ is

$$
\dot{\eta}=R_{A}^{-1} \omega \quad \rightarrow(4)
$$

Where

$$
R_{A}^{-1}=\left[\begin{array}{ccc}
1 & \sin \phi \tan \theta & \cos \phi \tan \theta \\
0 & \cos \phi & -\sin \phi \\
0 & \frac{\sin \phi}{\cos \theta} & \frac{\cos \phi}{\cos \theta}
\end{array}\right] \rightarrow(5)
$$

The translational dynamics of the hexacopter is described below.

In the earth fixed inertial frame, Newton's second law can be applied.

$$
F^{\varepsilon}=m \bar{\xi}=m a^{\varepsilon} \rightarrow(6)
$$

Where the time derivate is with respect to the inertial frame and $\boldsymbol{a}^{\boldsymbol{\varepsilon}}$ is the acceleration of the body fixed frame expressed in the inertial frame, $\mathrm{m}$ is the mass of the body which is constant and $\boldsymbol{F}^{\varepsilon}$ is sum of all external forces applied to the body expressed in the inertial frame. However, it would be convenient to express the dynamics in the body fixed frame. By using the rotational matrix from equation (2) a change of basis can be obtained and the expression becomes

$$
R_{L} F^{B}=m R_{L} a^{B}=m R_{L}\left(\frac{d V}{d t}\right)_{\varepsilon} \rightarrow(7)
$$

Where the time derivate is still with respect to the inertial frame and $\mathrm{V}$ is the velocity of the body fixed frame expressed in the body fixed frame. Computing that derivative is rather cumbersome, but using a well-known relation, sometimes called the transport theorem, yields

$$
F^{B}=m\left(\left(\frac{d V}{d t}\right)_{B}+\omega V\right) \rightarrow(8)
$$

Let the time derivative with respect to the body fixed frame be denoted by a dot, and then the final equation of the translational dynamics expressed in the body fixed frame is

$$
F^{B}=m V+\omega \times m V \rightarrow(9)
$$

The rotational dynamics of the hexacopter is described below

Rotational dynamics of a hexacopter is described using Euler's second axiom is

$$
M^{\varepsilon}=L^{\varepsilon} \rightarrow(10)
$$

Where the time derivative is with respect to the inertial frame, $\boldsymbol{L}^{\boldsymbol{\varepsilon}}$ is the angular momentum of the body expressed in the earth frame and $\boldsymbol{M}^{\varepsilon}$ is the sum of all external torques applied to the body expressed in the earth frame. The quantities can be expressed in body fixed frame by applying the rotational matrix for angular velocities from equation (4)

$$
R_{A} M^{B}=R_{A} \dot{L}^{B} \rightarrow(11)
$$

Where $\boldsymbol{L}=\boldsymbol{J} \boldsymbol{\omega}$ and $\boldsymbol{J}$ is the inertia matrix expressed in the body frame. Differentiating $J$ with respect to the earth frame is rather difficult since it will be time dependent. Instead using the transport theorem, the expression become

$$
M^{B}=\left(\frac{d J \omega}{d t}\right)_{B}+\omega \times J \omega \rightarrow(12)
$$

Since inertia matrix is time invariant the final equation is

$$
M^{B}=J \dot{\omega}+\omega \times J \omega \rightarrow(13)
$$

The external forces and torques acting on the body are described below

The relation in equations (9) and (13) are general equations of motion for a rigid body, these equations applying on the hexacopter system by finding different components of the external forces $\mathrm{F}$ and $\mathrm{M}$.

The forces and torques acting on the hexacopter are gravity, air friction aerodynamic forces and torques produced by the propellers and the gyroscopic effects from the rotation of the propellers. The torque caused by the angular acceleration of the propellers has been neglected. All the quantities are expressed in body frame.

\section{a. Gravity}

First of is the gravity which is the only force or torque which is naturally expressed in the earth frame. The gravitational force is acting on the hexacopter's center of gravity according to Euler's first axiom and is directed along the $z_{\mathrm{E}^{-}}$ axis. In the body fixed frame the contribution of the gravitational force $\boldsymbol{F}_{\boldsymbol{G}}^{\boldsymbol{B}}$ is

$$
F_{G}^{B}=R_{L}\left[\begin{array}{c}
0 \\
0 \\
m g
\end{array}\right]=m g\left[\begin{array}{c}
\sin \theta \\
\sin \phi \cos \theta \\
\cos \phi \cos \theta
\end{array}\right] \rightarrow(14)
$$

Where $\boldsymbol{g}$ is acceleration due to gravity. 


\section{b. Thrust and Torque from Propellers}

The second contribution is the thrust produced by the aerodynamics of the propellers and reaction torque from the rotation of the rotors. The sum of the thrust from the different propellers is total lift force and is always directed along the negative $z^{\mathrm{B}}$-axis. If the thrust from Propellers $i$ is denoted $\boldsymbol{T}_{\boldsymbol{i}}$ then the lift force $\boldsymbol{F}_{\boldsymbol{\omega}}$,

$$
F_{\omega}=T_{1}+T_{2}+T_{3}+T_{4}+T_{5}+T_{6} \rightarrow(15)
$$

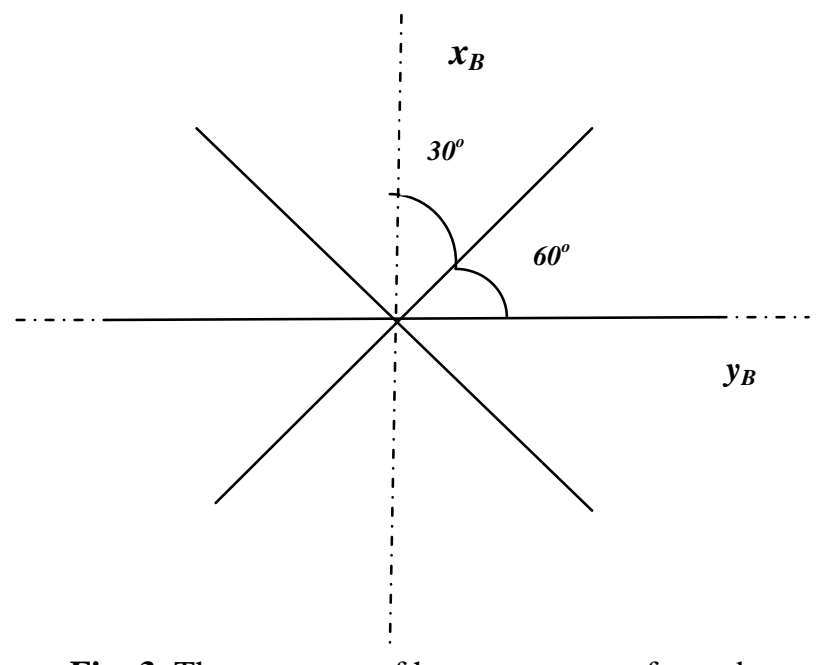

Fig -3: The geometry of hexacopter seen from above

Since the sources of thrust, that is the propellers are not located in the center of gravity, they will create torques around the different axes of rotation. With some basic geometry seen in fig: 1 it is easy to find the produced torque. Around the $x^{\mathrm{B}}$-axis the torque $\boldsymbol{M}_{\boldsymbol{p}}$ from propeller thrust is

$$
M_{p}=-\frac{l}{2} T_{1}-l T_{2}-\frac{l}{2} T_{3}+\frac{l}{2} T_{4}+l T_{5}+\frac{l}{2} T_{6} \rightarrow(16)
$$

where $l$ is the length of each arm. Around $y^{\mathrm{B}}$ axis the torque is $\boldsymbol{M}_{\boldsymbol{q}}$

$$
M_{q}=\frac{\sqrt{3} l}{2} T_{1}-\frac{\sqrt{3} l}{2} T_{3}-\frac{\sqrt{3} l}{2} T_{4}+\frac{\sqrt{3} l}{2} T_{6} \rightarrow(17)
$$

The torque around the $z^{\mathrm{B}}$-axis is result of Newton's third law. If the reaction torque from propeller $i$ is called the total torque around the $z^{\mathrm{B}}$-axis denoted $\boldsymbol{M}_{\boldsymbol{r}}$ is

$$
M_{r}=-\tau_{1}+\tau_{2}-\tau_{3}+\tau_{4}-\tau_{5}+\tau_{6} \rightarrow(18)
$$

The reaction torque is produced by the two different sources. When the motors and propellers accelerate, they will exert a torque on the air frame. Also, pressing the propeller through the air creates friction. The thrust and aerodynamic torque produced by a propeller can be related to the rotational speed of the propeller blades. If the rotational speed of propeller $\boldsymbol{i}$ is denoted $\boldsymbol{\Omega}_{\boldsymbol{i}}$, then the generated thrust is

$$
T_{i}=K_{T} \Omega_{i}^{2} \rightarrow(19)
$$

Where $\boldsymbol{K}_{\boldsymbol{T}}$ is a propeller specific constant explained more thoroughly later. In the same way, the aerodynamic torque $\boldsymbol{Q}_{\boldsymbol{i}}$ of propeller $\boldsymbol{i}$ becomes

$$
Q_{i}=K_{Q} \Omega_{i}^{2}
$$

Where $\boldsymbol{K}_{\boldsymbol{Q}}$ is a propeller specific constant.

Now, the total torque $\boldsymbol{M}_{\boldsymbol{A}}$ from the aerodynamic effects of the propeller can be written as

$$
\begin{gathered}
M_{A} \\
\rightarrow\left(\begin{array}{c}
-\frac{l}{2} K_{T} \Omega_{1}^{2}-l \Omega_{2}^{2}-\frac{l}{2} K_{T} \Omega_{3}^{2}+\frac{l}{2} K_{T} \Omega_{4}^{2}+l \Omega_{5}^{2}+\frac{l}{2} K_{T} \Omega_{6}^{2} \\
\frac{\sqrt{3} l}{2} K_{T} \Omega_{1}^{2}-\frac{\sqrt{3} l}{2} K_{T} \Omega_{3}^{2}-\frac{\sqrt{3} l}{2} K_{T} \Omega_{4}^{2}+\frac{\sqrt{3} l}{2} K_{T} \Omega_{6}^{2} \\
-K_{Q} \Omega_{1}^{2}+K_{Q} \Omega_{2}^{2}-K_{Q} \Omega_{3}^{2}+K_{Q} \Omega_{4}^{2}+K_{Q} \Omega_{5}^{2}+K_{Q} \Omega_{6}^{2}
\end{array}\right]
\end{gathered}
$$

And the total force $\boldsymbol{F}_{\boldsymbol{A}}$ can be written as

$$
F_{A}=\left[\begin{array}{c}
0 \\
0 \\
-K_{T}\left(\Omega_{1}^{2}+\Omega_{2}^{2}+\Omega_{3}^{2}+\Omega_{4}^{2}+\Omega_{5}^{2}+\Omega_{6}^{2}\right)
\end{array}\right] \rightarrow(22)
$$

\section{c. Gyroscopic Effects from Propellers}

If the rotation around the shaft is the spin and the rotation of the airframe is the precession, the gyroscopic torque produced by the propeller can be found. The spin quantity is already defined as $\boldsymbol{\Omega}_{\boldsymbol{i}}$.

The Gyroscopic torque $\boldsymbol{M}_{\boldsymbol{G}_{\boldsymbol{i}}}$ from propeller $\boldsymbol{i}$ is

$$
M_{G_{i}}=\omega \times J_{P}\left[\begin{array}{c}
0 \\
0 \\
(-1)^{I} \Omega_{i}
\end{array}\right] \rightarrow(23)
$$

Where $\boldsymbol{J}_{\boldsymbol{P}}$ is the inertia matrix of the propeller around the propeller axis and the factor $(-\mathbf{1})^{\boldsymbol{i}}$ come from the fact that the propellers rotate in opposite direction. Using the fact that the inertia matrix is a diagonal matrix the expression can be simplified to $\boldsymbol{M}_{\boldsymbol{G}_{\boldsymbol{i}}}=\boldsymbol{\omega} \times\left[\begin{array}{c}\mathbf{0} \\ \mathbf{0} \\ \boldsymbol{J}_{\boldsymbol{P}, \mathrm{ZZ}}(-\mathbf{1})^{\boldsymbol{I}} \boldsymbol{\Omega}_{\boldsymbol{i}}\end{array}\right]$

$$
=\left[\begin{array}{c}
q J_{P, Z Z}(-1)^{i} \Omega_{i} \\
-p J_{P, Z Z}(-1)^{i} \Omega_{i} \\
0
\end{array}\right] \rightarrow(24)
$$

Summing up all propellers, the total gyroscopic torque is

$$
M_{G}=\left[\begin{array}{c}
q J_{P, Z Z}\left(-\Omega_{1}+\Omega_{2}-\Omega_{3}+\Omega_{4}-\Omega_{5}+\Omega_{6}\right) \\
-p J_{P, Z Z}\left(-\Omega_{1}+\Omega_{2}-\Omega_{3}+\Omega_{4}-\Omega_{5}+\Omega_{6}\right) \\
0 \\
\rightarrow(25)
\end{array}\right]
$$




\section{d. Air Friction}

The air frames movement through the air will cause friction. Because of the shape of the air frame, the air friction is assumed to be low and a simple model is sufficient. The model of the air friction is given by

$$
\begin{gathered}
\boldsymbol{F}_{R}=-\boldsymbol{A}_{\boldsymbol{T}} \cdot \boldsymbol{V} \rightarrow(26) \\
\boldsymbol{M}_{\boldsymbol{R}}=-\boldsymbol{A}_{\boldsymbol{R}} \cdot \boldsymbol{\omega} \rightarrow(27)
\end{gathered}
$$

Where $\boldsymbol{A}_{\boldsymbol{T}}$ and $\boldsymbol{A}_{\boldsymbol{T}}$ are diagonal matrices with diagonal elements $\boldsymbol{a}_{\boldsymbol{T}}$ and $\boldsymbol{a}_{\boldsymbol{r}}$ respectively.

\subsection{Thruster Model}

The thruster model can be separated into two subsystems they are the propeller and electric motor. The propeller converts rotational speed into thrust and the electrical motor converse voltage into rotational speed. The propeller model is described below

The basic propeller model which can generate thrust $\boldsymbol{T}$ is

$$
T=C_{T} \rho r_{P}^{4} \pi \Omega^{2} \rightarrow(28)
$$

where $\boldsymbol{\rho}$ is the air density, $\boldsymbol{r}_{\boldsymbol{P}}$ is the propeller radius and $\boldsymbol{C}_{\boldsymbol{T}}$ is the thrust coefficient of the propeller. Similarly, the aerodynamic torque $\boldsymbol{Q}$ is expressed as

$$
Q=C_{Q} \rho r_{P}^{5} \pi \Omega^{2}
$$

Where $\boldsymbol{C}_{\boldsymbol{Q}}$ is the propeller torque constant.

The electrical model of the hexacopter can be expressed as In electrical motor model the ESCs receives a PWM signal which it decodes to a desired rotor speed. The interesting part of the dynamics of the rotor is the time constant and the PWM to rotor speed relation. Because of that, a PWM to voltage mapping and a classic model of a DC motor is considered. Hence the inductance and the friction caused by the moving parts of the motor are zero the model becomes a first order model which is considered enough. The model is defined by

$$
\begin{gathered}
I=\frac{V_{i n}-K_{v} \Omega}{R} \rightarrow(29) \\
\Omega=\frac{\boldsymbol{K}_{t}}{I_{\boldsymbol{P}}}+\boldsymbol{I}-\frac{\boldsymbol{\tau}}{\boldsymbol{I}_{\boldsymbol{P}}} \rightarrow(30) \\
\tau=\boldsymbol{Q} \rightarrow(31)
\end{gathered}
$$

Where $\boldsymbol{I}$ is the current, $\boldsymbol{V}_{\boldsymbol{i n}}$ is the voltage input, $\boldsymbol{K}_{\boldsymbol{v}}$ and $\boldsymbol{K}_{\boldsymbol{t}}$ are speed and torque constants respectively, is the motor speed, $\boldsymbol{\Omega}$ is the motor speed, $\boldsymbol{\tau}$ is the reaction torque and $\boldsymbol{Q}$ is the aerodynamic torque.

\subsection{System Model}

Combining the first derivative of the position equation (2), the first derivative of the orientation equation (4), the translational dynamics equation (9), the rotational dynamics equation (13), and the external forces equations (14), (21), (22) and(25) the final model becomes

$$
\begin{gathered}
\dot{\xi}=R_{L}^{T} \boldsymbol{V} \rightarrow(32) \\
\boldsymbol{m} \dot{\boldsymbol{V}}=-\boldsymbol{\omega} \times \boldsymbol{m} \boldsymbol{V} \rightarrow(33) \\
\dot{\boldsymbol{\eta}}=\boldsymbol{R}_{A}^{T} \boldsymbol{\omega} \rightarrow(34)
\end{gathered}
$$

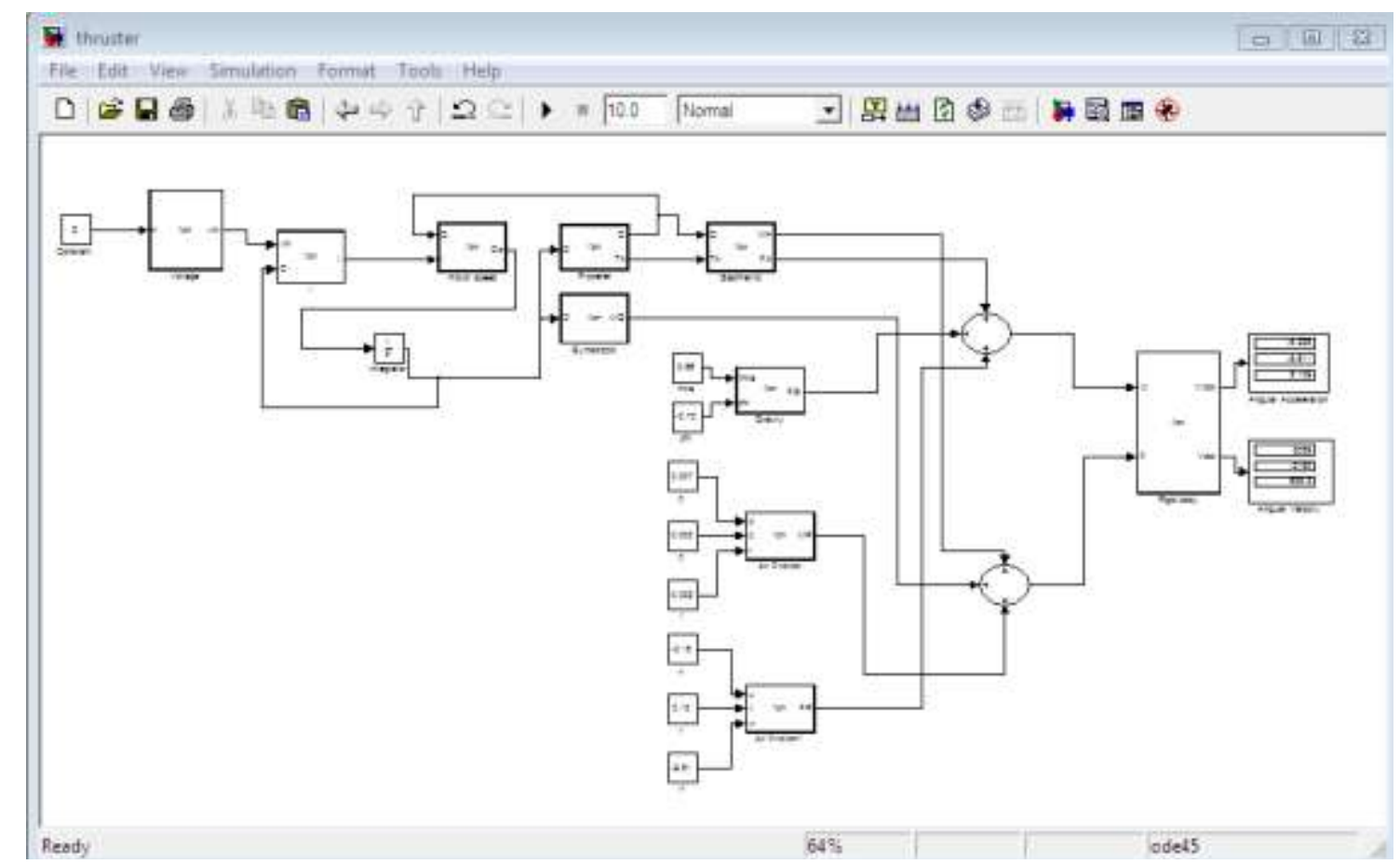

Fig -4: Flight dynamics on rigid body in simulink model 


\section{RESULT}

The Results after the autonomous flight have shown below

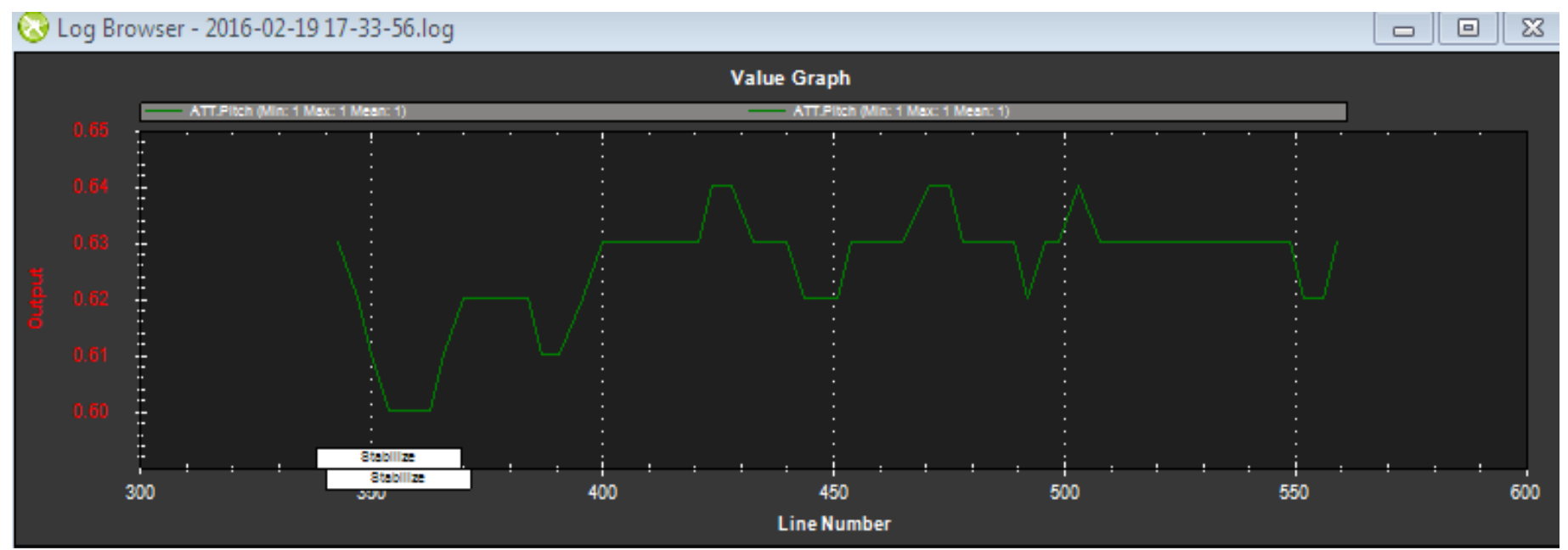

Fig -4: pitch result after autonomous flight

This is the behaviour of the copter during pitch operation during autonomous flight.

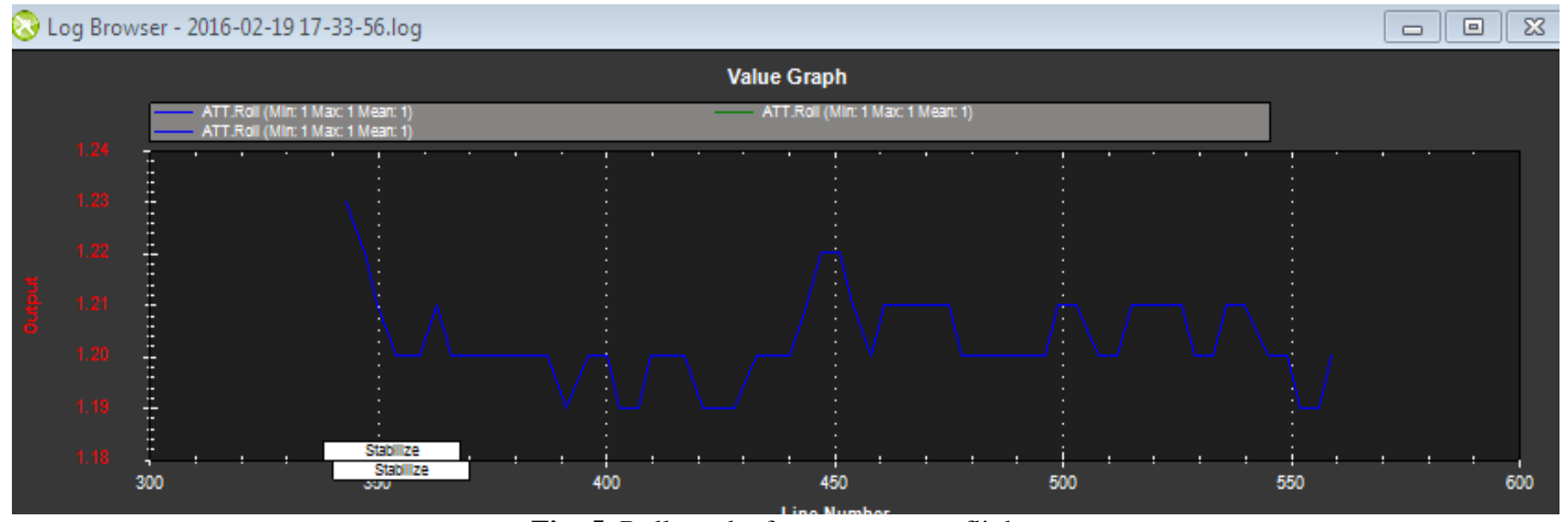

Fig -5: Roll result after autonomous flight

This is the behaviour of the copter during Roll operation during autonomous flight.

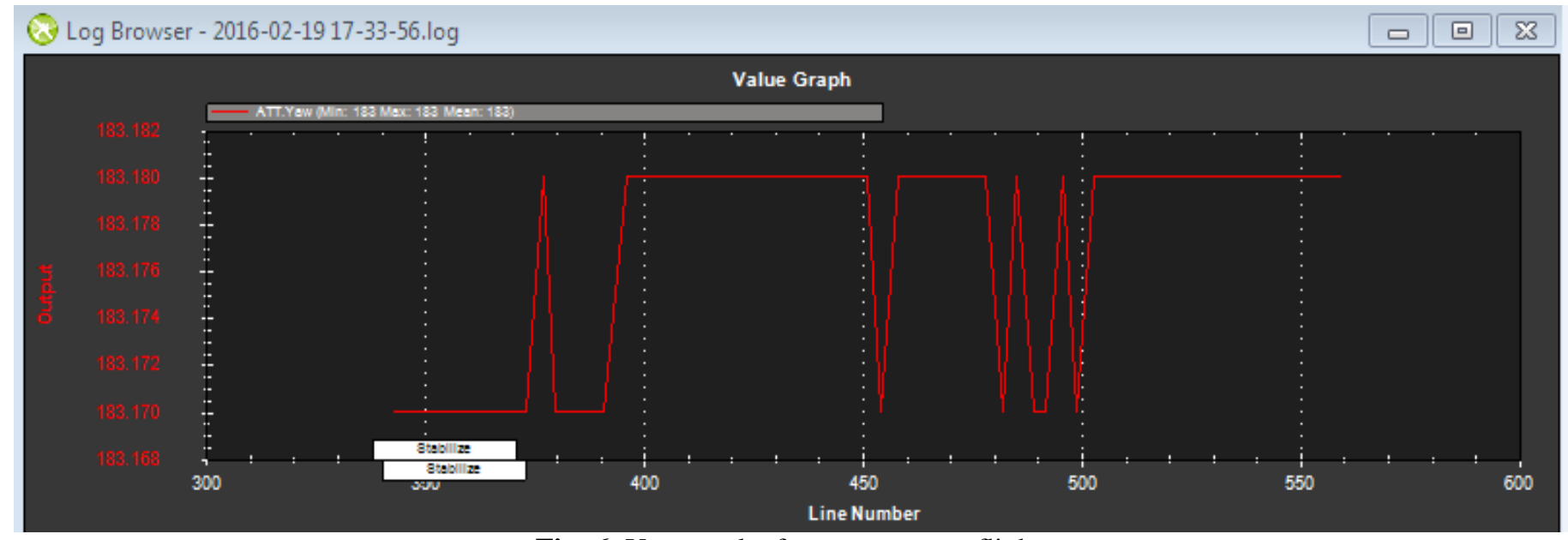

Fig -6: Yaw result after autonomous flight

This is the behaviour of the copter during Yaw operation during autonomous flight. 

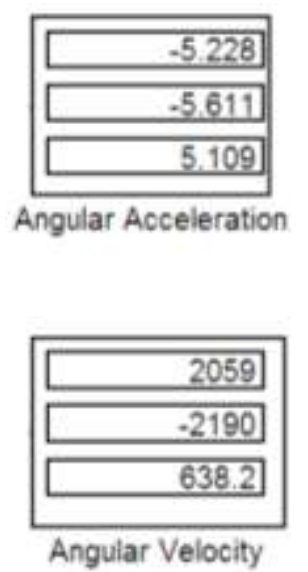

Fig -7: Orientation output of rigid body in simulink

\section{CONCLUSION}

Here in this paper the detailed overview of the flight dynamics are described, to determine the orientation of the hexacopter F550 in the form of angular acceleration and angular velocities by using log data generated by the copter while flying. The log data has used in external dynamics like Air friction, Gravity and the internal dynamics like thrust which are applying on a rigid body to measure the orientation of the rigid body model of the hexacopter. To Acquire the logdata we have performed a number of autonomous flights.

\section{REFERENCES}

[1] Hai Chen Xin-min Wang Yan Li Hai Chen Xin-min Wang Yan Li "A Survey of Autonomous Control for UAV", International Conference On Artificial Intelligence And Computational Intelligence (AICI 2009), Shanghai, Nov 2009, pp.267-271.

[2] Grenville Barnes, Walter Volkmann, Romeo Sherko, Kathrine Kelm, "Design and Testing of a UAV-based Cadastral Surveying and Mapping Methodology in Albania", In proceedings of annual world bank conference on land and poverty, Washington DC, Mar 2014.

[3] R. Aust, "Mission planner evaluation in the mission reconfigurable cockpit", In Proceedings of IEEE International conference on Aerospace and Electronics (NAECON 1996), May 1996, pp. 102-108.

[4] Jordan Cox, Derek Burdette, Vhristopher Shaffer, Farshid Zabihian, "Lighter than UAV', In proceedings of zone $i$ conference on industry involvement and interdisciplinary trends (ASEE 2014), university of Bridgeport, CT, USA, April 2014,

[5] V. Artale, C.L.R. Milazzo, A. Ricciardello, "Mathematical Modelling of Hexacopter", In proceedings of IEEE International Conference on Unmanned Aerial Systems(ICUAS 2013), Atlanta, GA, May 2013, pp.1043-1050.

[6] Ioan-Radu Morar, Ioan Nascu, "Model Specification of an unmanned Aerial Vehicle", In proceedings of IEEE International conference on Automation Quality and Testing Robotics (AQTR 2012), May 2012, pp. 591596.
[7] D. b. W. Ashfaq Ahmad Mian, "dynamic modeling and nonlinear control strategy for an underactuated quadrotor rotorcraft", Journal of Zhejiang University, SCIENCE A, vol. 9, iss. 4, jan-2008, pp. 539-545.

[8] A. Tayebi, S. McGilvray "Attitude Stabilization of a four-rotor aerial robot", In proceedings of $43^{\text {rd }}$ IEEE international conference on Decision and Control (CDC 2004), Dec 2004, Vol. 2, pp. 1216-1221. 